\title{
Dust content of core-collapse supernova hosts
}

\author{
A.-L. Melchior ${ }^{1,2}$ and F. Combes ${ }^{1}$ \\ 1 LERMA, Observatoire de Paris, CNRS, UMR8112, 61 avenue de l'Observatoire, 75014 Paris, France \\ e-mail: [A.L.Melchior; Francoise.Combes]@obspm. fr \\ ${ }^{2}$ Université Pierre et Marie Curie-Paris 6, 4 Place Jussieu, 75252 Paris Cedex 05, France
}

Received 1 August 2007 / Accepted 13 March 2008

\section{ABSTRACT}

\begin{abstract}
We study 13 core-collapse supernova (CCSN) host galaxies at $z=0.1-0.6$. Continuum observations at $250 \mathrm{GHz}$ were performed using MAMBO at the IRAM-30 m telescope. No source is detected and the error-weighted mean flux is $0.25 \pm 0.32 \mathrm{mJy}$. Upper limits on their dust masses are derived and the corresponding sample mean corresponds to $1.4 \pm 2.2 \times 10^{8} M_{\odot}$. These results are comparable to previous submillimetre observations of SN-Ia hosts completed by Farrah et al. and Clements et al. We conclude that CCSN hosts are not extreme sources at millimetre wavelengths, and as confirmed using the optical luminosities of a subset of our sample, they are typical of the local galaxy population.
\end{abstract}

Key words. stars: supernovae: general - galaxies: starburst - radio continuum: galaxies - galaxies: high-redshift galaxies: evolution - ISM: dust, extinction

\section{Introduction}

In the local universe, type-Ia supernovae (SN-Ia) are detected in host galaxies along the entire Hubble sequence, while the core-collapse supernovae (CCSN) are uncommon inside earlytype galaxies, which do not have gas. Star-formation activity is known to increase with redshift (e.g. Madau et al. 1996, 1998; Chary \& Elbaz 2001; Blain et al. 2002), while no evolution has yet been observed in supernova properties (e.g. Combes 2004). Submillimetre and millimetre (submm/mm) galaxies (SMG) account for a significant fraction of the submm/mm Cosmic Background (e.g. Hughes et al. 1998; Barger et al. 1999), and $50-70 \%$ have a radio counterpart, at the detection level of $100 \mu \mathrm{Jy}$. Most of these SMG are detected at $z \sim 2-3$ (e.g. Smail et al. 2000; Chapman et al. 2003a,b, 2005). The submm/mm fluxes thus correspond to the redshifted far-infrared (FIR) peak of the dust emission. Our current understanding of the FIR/radio correlation (Condon 1992) is that synchrotron emission detected at radio wavelengths is due to relativistic electrons accelerated by shocks during supernova explosions. Numerous works support this interpretation of the FIR/radio emission in terms of recent star formation activity (e.g. Boyle et al. 2007; Vlahakis et al. 2007), pointing towards a strong link between SMG and strong starbursts. Dunne et al. (2003) showed that CCSN are at least as important as stellar winds, which enrich our Galaxy with dust, and suggested that they could be the dominant source of dust at high redshift. The role of supernovae and CCSN should be important to these mechanisms. However, a number of difficulties prevent definite conclusions. In the optical, dust extinction biases the detection of CCSN in the core of strong starbursts (Mattila \& Meikle 2001): Mattila et al. (2007) detected, for the first time, a CCSN within a circumnuclear starburst, that experienced 40 mag. of extinction in $V$. There is also still no direct evidence that CCSN could be dust factories at high- $z$ (Meikle et al. 2007). The rates of radio supernovae (e.g. Lonsdale et al. 2006) and supernova remnants (e.g.
Lenc \& Tingay 2006) do not suffer optical extinction, but only probe nearby starbursts so far.

In the local universe, the strongest starbursts are found in interacting and merging galaxies (Sanders \& Mirabel 1996), while Conselice et al. (2003) suggested that two thirds of the SMG at $z>1$ are undergoing a major merger. These observations agree with theoretical hierarchical galaxy formation scenarios, even though all mergers do not trigger a strong starburst (e.g. Bergvall et al. 2003; di Matteo et al. 2007). The dust attenuation is expected to increase with the bolometric luminosity of the galaxies and their star formation rate, the evolution of the dust attenuation with redshift is still a matter of debate (e.g. Buat et al. 2007). Nevertheless, SMG observations suggest that these young galaxies contain a large fraction of gas (e.g. Neri et al. 2003; Greve et al. 2005; Tacconi et al. 2006), probably associated with a large amount of dust.

The variation with redshift of the mean extinction affecting hosts is a real concern for high- $z$ SN searches. Farrah et al. (2004) tentatively argue that the extinction in high- $z$ SN-Ia hosts may increase with redshift (see also e.g. Totani \& Kobayashi 1999). Selection effects are thought to favour hosts with less extinction (e.g. Commins 2004), since very extinguished supernovae are not detected in the actual surveys. Several SN-Ia surveys apply cuts to the $\left(A_{V} \leq 0.5-1\right)$ extinction (see e.g. Tonry et al. 2003; Riess et al. 2004, 2005, 2007), while moderate extinction is removed implicitly in the MLCS and $\Delta m_{15}$ reduction methods (Riess et al. 1996; Hamuy et al. 1996). There are indications that the distant SN-Ia might have a bluer colour than their local counterparts (Leibundgut 2001, and references therein). Chary et al. (2005) discovered, using Spitzer MIPS observations of the GOODS fields, that supernova host galaxies have a detection rate at $24 \mu \mathrm{m}$ that is a factor of 1.5 higher than the field galaxy population. Their study is based on 50 supernovae sampled up to $z \sim 1$ and surprisingly, they find similar properties for $\mathrm{SN}-\mathrm{Ia}$ and CCSN hosts. 
Table 1. List of the CCSN galaxies observed to detect 250GHz-MAMBO continuum at IRAM-30 m. (1): IAU designation; (2, 3): J2000 RA, Dec coordinates; (4): redshift of the host galaxy or the SN; (5): SN type; (6): detection magnitude of the SN; (7): IAU circular numbers.

\begin{tabular}{|c|c|c|c|c|c|c|}
\hline $\begin{array}{l}\text { Name } \\
(1) \\
\text { SN }\end{array}$ & $\begin{array}{l}\text { RA } \\
(2) \\
\text { J2000 }\end{array}$ & $\begin{array}{l}\text { Dec } \\
\text { (3) } \\
\text { J2000 }\end{array}$ & $\begin{array}{l}z \\
(4)\end{array}$ & $\begin{array}{l}\text { type } \\
\text { (5) }\end{array}$ & $\begin{array}{l}\text { Mag. } \\
\text { (6) }\end{array}$ & $\begin{array}{l}\text { IAUC } \\
\text { (7) }\end{array}$ \\
\hline 1995av & 020136.7 & +033855.2 & 0.30 & III & 20.1 & 6270 \\
\hline 1999fl & 023005.5 & +004452.6 & 0.3 & II & 24.9 & 7312 \\
\hline $2001 \mathrm{ek}^{a}$ & 023017.6 & +010356.4 & $0.25-0.40$ & IIP & 25.1 & 7719 \\
\hline 1999fp & 041502.5 & +042146.4 & 0.34 & II? & 24.1 & 7312 \\
\hline 2000ei & 041707.2 & +05 4553.1 & 0.60 & II? & 22.8 & 7516 \\
\hline $1997 \mathrm{ev}$ & 082420.2 & +035136.0 & 0.43 & II? & 23.0 & 6804 \\
\hline 1997ew & 082425.0 & +034908.0 & 0.59 & II/Ic? & 23.9 & 6804 \\
\hline 1998at & 105454.3 & -034410.0 & 0.40 & II & 23.8 & 6881 \\
\hline 2001ct & 132444.6 & +271527.2 & 0.45 & II? & 23.4 & 7649 \\
\hline $2002 \mathrm{~cm}$ & 135203.7 & -114308.5 & 0.087 & I & 22.3 & 7885 \\
\hline 2002du & 135318.3 & -113728.8 & 0.21 & II & 22.2 & 7929 \\
\hline 2001gl & 140116.6 & +05 1248.9 & 0.36 & $\mathrm{Ib} / \mathrm{c}$ & 23.7 & 7763 \\
\hline $2002 \mathrm{co}$ & 141053.0 & -114525.0 & 0.318 & II & 22.6 & 7885 \\
\hline
\end{tabular}

${ }^{a}$ We assume $z=0.25$ in Fig. 1.

Farrah et al. (2004) detected statistically at the $3 \sigma$ significance level the continuum emission at $350 \mathrm{GHz}$ of a sample of $16 \mathrm{SN}-\mathrm{Ia}$ hosts at $z=0.5$, while one submm strong source was detected directly. The authors interpreted this result as the signature of a slight increase of $25 \%-135 \%$ in the dust content of this galaxy sample, with respect to local normal galaxies studied by Rowan-Robinson (2003). However, one should note that the definition of a "normal galaxy" reference sample is one difficulty in this type of work, which imposes magnitude thresholds and requires infrared/ultraviolet detections.

We perform a study of the $1.2 \mathrm{~mm}$ continuum for a sample of 13 CCSN. In Sect. 2, the observations performed at the IRAM-30 m telescope are presented with a description of the data reduction using the MOPSI software. In Sect. 3, we discuss our results.

Throughout this paper, we adopt a flat cosmology with $\Omega_{\mathrm{m}}=$ $0.3, \Omega_{\Lambda}=0.7$ and $H_{0}=70 \mathrm{~km} \mathrm{~s}^{-1} \mathrm{Mpc}^{-1}$.

\section{Observations and data reduction}

From the IAU list, we selected CCSN host galaxies with a redshift identification available in the range $0.1<z<1$ and a secure core-collapse type. The IAU circulars that correspond to each selected CCSN are indicated in Table 1.

Millimetre continuum measurements were made during the Summer-2002 and Winter-2003 Pool observations on the MAMBO-1 (37-channels) and MAMBO-2 (117-channels) arrays installed on the IRAM-30 m telescope (IRAM ${ }^{1}$, Pico Veleta, Spain). The beam size (HPBW) was 11". Each of the CCSN host galaxies listed in Table 1 were observed at $250 \mathrm{GHz}$ using the on-off mode in the most sensitive channel (resp. no 1 and 20). To account for variations in the sky brightness, we used standard chopping of the secondary mirror of the telescope between the on-source position and a position $\pm 33^{\prime}$ away in azimuth, at a rate of $2 \mathrm{~Hz}$. The telescope was nodded every $10 \mathrm{~s}$ such that the previous "off" position became the "on" position, to subtract background asymmetries between the two wobbler positions. Measurements of the sky opacity, sky dips, were taken

1 IRAM is a joint organisation founded by the German Max-PlanckSociety, the French CNRS and the Spanish National Geographical Institute. approximately every two hours, while calibration sources were regularly monitored. The pointing was checked every $30-60 \mathrm{~min}$ depending on the stability of the observing conditions. The focus was regularly checked every $1-2 \mathrm{~h}$.

The data were reduced using the MOPSI software (Zylka 1998) following standard procedures. A visual inspection of each scan had first been performed to remove very noisy subscans. The skynoise reduction used measurements from the six channels surrounding the photometric channel. Extinction correction was performed relying on a linear interpolation between sky dips taken before and after each set of observations, or the closest value.

\section{Results and discussion}

\subsection{Detection rate}

As shown in Table 2, we were unable to detect any of the $13 \mathrm{CCSN}$ host galaxies that we observed. We compute the errorweighted mean flux of the entire sample and find $0.25 \pm 0.32 \mathrm{mJy}$. We reach a sensitivity comparable to that of the sample of Farrah et al. (2004) $(1.55 \pm 0.31 \mathrm{mJy})$. However, we do not find any trace of signal. The detection of Farrah et al. (2004) was dominated by 2 sources (SN1997ey and SN2000eh hosts) and hence very sensitive to statistical fluctuations. Furthermore, we showed in Melchior \& Combes (2007) that the strongest of these 2 sources (SN1997ey host) has no significant gas content given its submillimetre flux: this flux might be due to a background source. Excluding this source, Farrah et al. (2004) found an error-weighted mean flux of their 16 galaxies of $1.01 \pm$ $0.33 \mathrm{mJy}$. Removing the second source, which was detected only at $850 \mu \mathrm{m}$ with $3 \sigma$ significance level, their mean flux reduced to $0.84 \pm 0.40 \mathrm{mJy}$. Clements et al. (2005) subsequently extended the $\mathrm{SN}$-Ia host sample to 31 galaxies and found a less significant signal $(0.44 \pm 0.22 \mathrm{mJy})$. Hence, we conclude that our CCSN host measurements are consistent with the SN-Ia host findings. However, we note that at a given significance level of sensitivity, the SCUBA measurements probe the thermal emission of galaxies far deeper than our observations, because the 850 -to- $1200 \mu \mathrm{m}$ flux ratio is expected to be about three. 
Table 2. List of the CCSN galaxies observed for $250 \mathrm{GHz}-\mathrm{MAMBO}$ continuum at IRAM-30 m. (1): IAU designation; (2): zenith optical depth of the atmosphere at $250 \mathrm{GHz}$; (3): measured flux and $1 \sigma$ error of the sources; (4): dust mass assuming $T_{\mathrm{d}}=15 \mathrm{~K}$; (5): dust mass assuming $T_{\mathrm{d}}=20 \mathrm{~K}$; (6): date of the observation; (7): integration time in minutes; (8): bolometer used: MAMBO-1 (37-channels) or MAMBO-2 (117-channels).

\begin{tabular}{cccccccc}
\hline \hline $\begin{array}{c}\text { Name } \\
(1)\end{array}$ & $\begin{array}{c}\tau_{250 \mathrm{GHz}} \\
(2)\end{array}$ & $\begin{array}{c}S\left(v_{250 \mathrm{GHz}}\right) \\
(3)\end{array}$ & $\begin{array}{c}M_{\text {dust }}^{15 \mathrm{~K}} \\
(4)\end{array}$ & $\begin{array}{c}M_{\text {dust }}^{20 \mathrm{~K}} \\
(5)\end{array}$ & $\begin{array}{c}\text { date } \\
(6)\end{array}$ & $\begin{array}{c}\text { IT } \\
(7)\end{array}$ & $\begin{array}{c}\text { Bolo } \\
(8)\end{array}$ \\
SN & & $\mathrm{mJy}$ & $10^{9} M_{\odot}$ & $10^{9} M_{\odot}$ & & & \\
\hline $1995 \mathrm{av}$ & $0.54-0.66$ & $0.96 \pm 1.25$ & $0.39 \pm 0.60$ & $0.25 \pm 0.39$ & $19 / 10 / 02$ & 36 & 37 \\
$1999 \mathrm{fl}$ & $0.58-0.62$ & $-2.63 \pm 1.89$ & $-2.86 \pm 1.61$ & $-1.77 \pm 1.00$ & $19 / 10 / 02$ & 18 & 37 \\
$2001 \mathrm{ek}^{a}$ & $0.50-0.54$ & $-0.22 \pm 1.48$ & $-0.18 \pm 0.89$ & $-0.12 \pm 0.56$ & $15 / 03 / 03$ & 26 & 117 \\
$1999 \mathrm{fp}$ & $0.39-0.89$ & $0.61 \pm 0.53$ & $0.05 \pm 0.10$ & $0.03 \pm 0.07$ & $05,11 / 10 / 02$ & 132 & 37 \\
$2000 \mathrm{ei}$ & $0.50-0.52$ & $-2.02 \pm 1.48$ & $-0.64 \pm 0.41$ & $-0.42 \pm 0.27$ & $01 / 03 / 03$ & 25 & 117 \\
$1997 \mathrm{ev}$ & $0.33-0.72$ & $0.33 \pm 0.59$ & $0.21 \pm 0.83$ & $0.14 \pm 0.53$ & $05,11 / 10 / 02$ & 93 & 37 \\
$1997 \mathrm{ew}$ & $0.27-0.39$ & $1.64 \pm 1.05$ & $0.91 \pm 0.81$ & $0.59 \pm 0.52$ & $31 / 10,01 / 11 / 02$ & 53 & 117 \\
$1998 \mathrm{at}$ & $0.30-0.35$ & $-0.98 \pm 1.10$ & $-0.51 \pm 0.97$ & $-0.33 \pm 0.63$ & $01 / 11 / 02$ & 48 & 117 \\
$2001 \mathrm{ct}$ & $0.29-0.43$ & $0.22 \pm 1.07$ & $0.13 \pm 0.32$ & $0.08 \pm 0.21$ & $10-11 / 02 / 03$ & 43 & 117 \\
$2002 \mathrm{~cm}$ & $0.45-0.53$ & $1.54 \pm 1.31$ & $1.12 \pm 0.80$ & $0.72 \pm 0.51$ & $02,10 / 02 / 03$ & 32 & 117 \\
$2002 \mathrm{du}$ & $0.48-0.57$ & $-0.67 \pm 1.30$ & $-0.53 \pm 0.47$ & $-0.34 \pm 0.30$ & $02,10 / 02 / 03$ & 39 & 117 \\
$2001 \mathrm{gl}$ & $0.37-0.55$ & $-0.02 \pm 0.84$ & $-0.02 \pm 1.12$ & $-0.01 \pm 0.70$ & $02,11 / 02 / 2003$ & 32 & 117 \\
& & & & & $13 / 03 / 03$ & 26 & 117 \\
& & & & & $01 / 11 / 02$ & 14 & 117 \\
$2002 \mathrm{co}$ & $0.53-0.55$ & $0.77 \pm 1.46$ & $0.40 \pm 0.64$ & $0.26 \pm 0.42$ & $02,11 / 02 / 03$ & 32 & 117 \\
\hline${ }^{a}$ We assume $z=0.25$ & & & & & &
\end{tabular}

\subsection{Dust masses and comparison}

The derivation of dust masses using $\mathrm{mm} / \mathrm{submm}$ continuum measurements depends on the complex parameters that characterise the dust. They were first provided by Hildebrand (1983), who used reflection nebulae data. Large scatters in the data affect the grain emissivity $Q(\lambda)$, the grain radius $a$, and the material density $\rho$, as studied subsequently by various authors (e.g. Draine \& Lee 1984; Casey 1991). Initial analyses were based on $100 / 250 \mu \mathrm{m}$ measurements, which probe the hot-dust component and tend to underestimate the total dust mass. James et al. (2002) proposed a new method to determine the dust mass-absorption coefficient $\kappa_{\mathrm{d}}(850 \mu \mathrm{m})=3 Q(850 \mu \mathrm{m}) /(4 a \rho)$, which assumed that the fraction of metals within the interstellar medium of a galaxy, bound up in dust, was constant. They provide arguments in favour of this assumption and estimate $\kappa_{\mathrm{d}}(850 \mu \mathrm{m})=0.07 \pm 0.02 \mathrm{~m}^{2} \mathrm{~kg}^{-1}$. This coefficient can be extrapolated to lower (or higher) frequencies using the emissivity index $\beta$, which is usually taken to be in the range $\beta \sim 1-2$ : $\kappa_{\mathrm{d}}(\lambda) \propto \nu^{\beta}$. Following Dunne et al. (2001), we considered $\beta=2$ and assumed optically-thin dust emission. The dust mass $M_{\text {dust }}$ was determined using the formula:

$M_{\text {dust }}=\frac{S\left(v_{\mathrm{obs}}\right) D_{\mathrm{L}}^{2}}{(1+z) \kappa_{\mathrm{d}}\left(v_{\mathrm{e}}\right) B\left(v_{\mathrm{e}}, T_{\mathrm{d}}\right)}$

where $v_{\mathrm{e}}$ and $v_{\mathrm{obs}}$ are the frequencies at which the radiation is emitted and observed, $T_{\mathrm{d}}$ is the dust temperature, $D_{\mathrm{L}}$ is the luminosity distance of the source, which was computed for a flat cosmology with a cosmological constant according to Pen (1999), $S\left(v_{\mathrm{obs}}\right)$ is the observed flux, and $B\left(v_{\mathrm{e}}, T_{\mathrm{d}}\right)$ is the Planck function.

Using Eq. (1) and the previous parameters, we converted our $250 \mathrm{GHz}$ continuum measurements into dust-mass upper limits and, in Fig. 1, superimpose them on various $(350 \mathrm{GHz}-$ based) dust-mass estimates derived in this redshift range. We note that, as shown by Seaquist et al. (2004) for the SLUGS data (Dunne et al. 2000), the estimates based on $350-\mathrm{GHz}$ data are overestimated by $25-38 \%$ due to contamination by the $\mathrm{CO}(3-2)$ line, which might be counter-balanced by an increased sensitivity to hot-dust components. We display the various dust-mass estimates obtained for $z<1.5$. The full bullets correspond to dust mass $M_{\mathrm{d}}^{\text {cold }}$ obtained for IRAS Bright

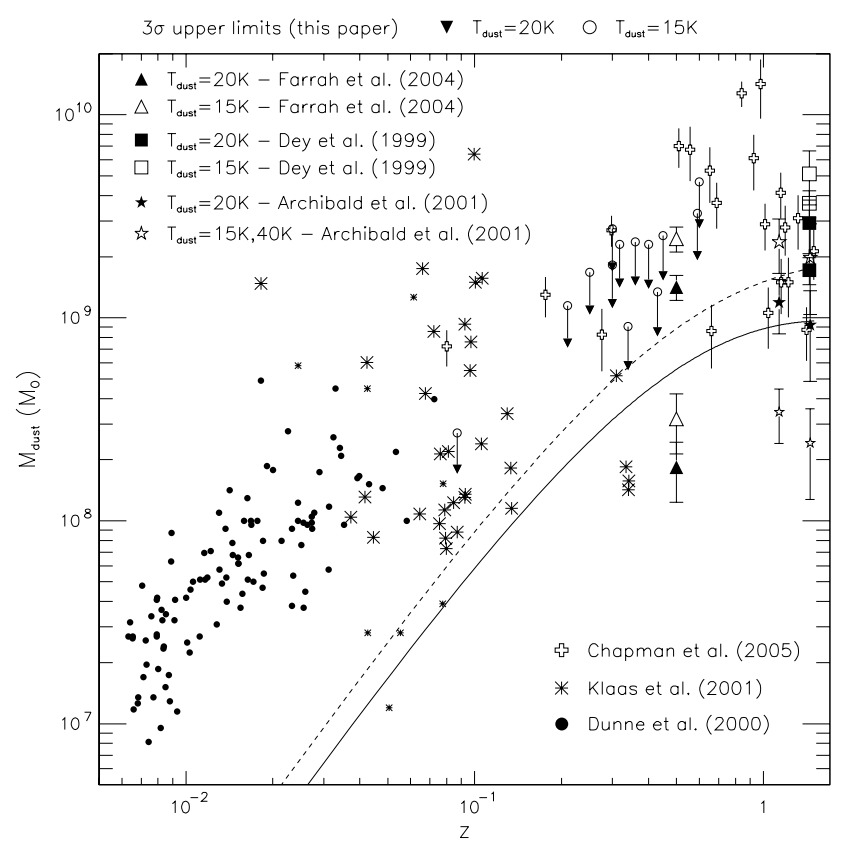

Fig. 1. Dust mass-redshift diagram. Upper limits obtained for the dust mass of CCSN hosts are superimposed on dust-mass estimates derived for other galaxies in this redshift range. We derive from our $250 \mathrm{GHz}$ measurements (provided in Table 1$)$ upper values $(3 \sigma)$ of the corresponding dust masses for $T_{\text {dust }}=20 \mathrm{~K}$ (filled inverted triangle symbols) and $T_{\text {dust }}=15 \mathrm{~K}$ (open circle symbols). Lines connect these 2 upper values. The other points correspond to dust masses derived from $350-\mathrm{GHz}$ (detected) fluxes (assuming $T_{\text {dust }}=20 \mathrm{~K}$ and $T_{\text {dust }}=15 \mathrm{~K}$ when indicated so). The upright triangle symbols correspond to the SN-Ia host detected by Farrah et al. (2004), and the mean flux derived from the rest of their SN-Ia host sample at $z=0.5$. The full (resp. dashed) line corresponds to a $1 \mathrm{mJy}(1 \sigma)$ sensitivity at $1.2 \mathrm{~mm}$ for $T_{\mathrm{d}} \sim 20 \mathrm{~K}$ (resp. $T_{\mathrm{d}} \sim 15 \mathrm{~K}$ ). See text for more details.

Galaxies $\left(L_{\mathrm{IR}}<10^{12} L_{\odot}\right)$ with a two-component-temperature fit to the data, assuming a cold component with $T_{\mathrm{d}}=20 \mathrm{~K}$ and $\beta=2$ (Dunne et al. 2000). The asterisk symbols provide the dust masses based on a multiple modified blackbody 
Table 3. Optical magnitude and intrinsic luminosity for a subset of the CCSN sample. The ugriz magnitudes were retrieved from the SDSS/DR6 database. The rest-frame B luminosities $\left(L_{\text {rest }}^{\mathrm{B}}\right)$ were computed using the known redshifts.

\begin{tabular}{lllllll}
\hline \hline $\begin{array}{l}\text { Name } \\
(1)\end{array}$ & $u$ & $g$ & $r$ & $i$ & $z$ & $L_{\text {rest }}^{\mathrm{B}}$ \\
units & mag & $(3)$ & $(4)$ & $(5)$ & $(6)$ & $(7)$ \\
\hline sn1999fl & $25.64 \pm 1.07$ & $22.63 \pm 0.20$ & $21.53 \pm 0.11$ & $20.62 \pm 0.07$ & $20.14 \pm 0.15$ & 0.5 \\
sn2001ek & $23.69 \pm 1.79$ & $23.33 \pm 0.48$ & $21.59 \pm 0.16$ & $20.70 \pm 0.11$ & $19.93 \pm 0.19$ & $0.6-2.5$ \\
sn1997ev & $21.91 \pm 0.35$ & $21.38 \pm 0.08$ & $20.38 \pm 0.05$ & $19.93 \pm 0.06$ & $19.42 \pm 0.14$ & 9.2 \\
sn1997ew & $22.48 \pm 0.49$ & $20.31 \pm 0.03$ & $18.93 \pm 0.01$ & $18.43 \pm 0.01$ & $18.06 \pm 0.03$ & 87 \\
sn2001ct & $23.41 \pm 1.08$ & $23.91 \pm 0.71$ & $22.11 \pm 0.24$ & $21.89 \pm 0.37$ & $22.34 \pm 1.27$ & 2.0 \\
sn2001gl & $22.65 \pm 0.36$ & $19.98 \pm 0.02$ & $18.61 \pm 0.01$ & $17.94 \pm 0.01$ & $17.59 \pm 0.01$ & 28 \\
\hline
\end{tabular}

Note: we consider $L_{*}^{\mathrm{B}}=2.3 \times 10^{9} L_{\odot}=1.9 \times 10^{10} L_{\odot}^{\mathrm{B}}$.

with $\beta=2$ for bright ultraluminous infrared galaxies (Klaas et al. 2001). The largest and smallest asterisk symbols correspond to galaxies with $L_{\mathrm{IR}}>10^{12} L_{\odot}$ and $L_{\mathrm{IR}} \leq 10^{12} L_{\odot}$, respectively. The crosses correspond to submillimetre galaxies from Chapman et al. (2005). For the remaining data sets, we derive dust masses using $350 \mathrm{GHz}$ flux measurements. The star symbols correspond to radio-galaxies (Archibald et al. 2001) for which the synchrotron emission was removed from the $350-\mathrm{GHz}$ fluxes. We note that the small-size star symbols refer to $T_{\text {dust }}=40 \mathrm{~K}$, which were favoured by these authors. The square symbols correspond to the galaxy HR10 (Dey et al. 1999). The upright triangle symbols indicate the SN-Ia hosts detections of Farrah et al. (2004).

Figure 1 shows that our upper limits are consistent with other data detected at $z<1.5$. We conclude that no galaxy in our sample of CCSN hosts contains a very large amount of dust $\left(M_{\text {dust }}>10^{9} M_{\odot}\right)$. The error-weighted mean flux corresponds to a dust mass of $1.4 \pm 2.2 \times 10^{8} M_{\odot}$, which is comparable with the Farrah et al. (2004) sample and the upper distribution of the local SLUGS sources of Dunne et al. (2000).

\subsection{Optical fluxes}

A subset (6/13) of our galaxies were observed by the SDSS. We retrieve the corresponding ugriz photometry and derive the intrinsic B luminosity as displayed in Table 3. We can clearly see the diversity of host galaxies in our sample: very massive systems $\left(87 L_{*}^{\mathrm{B}}\right)$ as well as normal galaxies $\left(0.5-2 L_{*}^{\mathrm{B}}\right)$ are present, with an average B luminosity of $21 L_{*}^{\mathrm{B}}$. This simply reflects the fact that these CCSN are detected in all types of galaxies containing gas and obeying the selection criteria.

\section{Conclusion}

We have observed 13 high-z CCSN host galaxies at $250 \mathrm{GHz}$ and detected no signal. We can exclude that individual galaxies contain more than about $10^{9} M_{\odot}$ of dust, while the error-weighted average dust mass is $1.4 \pm 2.2 \times 10^{8} M_{\odot}$ for the entire sample. Our results are compatible with those of Farrah et al. (2004) only if the strong source detected by these authors is excluded, while we find good agreement with the results of Clements et al. (2005). The B luminosities of a subset of our sample demonstrate the significant diversity of the host galaxies. This study shows that CCSN host galaxies are typical of galaxies observed in the local Universe (e.g. Kauffmann et al. 2003) and do not belong to the brightest SMG population.

Acknowledgements. We are very grateful to the anonymous referee for his valuable and detailled comments. We thank Carl Pennypacker who motivated this work during his sabbatical leave at LERMA in 2003. A.L.M. kindly acknowledges Axel Weiss for his help during the Pool observations at IRAM, and Robert Zylka for his efficient introduction to the MOPSI software. A.L.M. thanks J.-F. Lestrade for helpful discussions.

Photometry of 6 galaxies has been retrieved from SDSS/Data Release 6 . Funding for the SDSS and SDSS-II has been provided by the Alfred P. Sloan Foundation, the Participating Institutions, the National Science Foundation, the US Department of Energy, the National Aeronautics and Space Administration, the Japanese Monbukagakusho, the Max Planck Society, and the Higher Education Funding Council for England. The SDSS Web Site is http://www . sdss.org/. The SDSS is managed by the Astrophysical Research Consortium for the Participating Institutions. The Participating Institutions are the American Museum of Natural History, Astrophysical Institute Potsdam, University of Basel, University of Cambridge, Case Western Reserve University, University of Chicago, Drexel University, Fermilab, the Institute for Advanced Study, the Japan Participation Group, Johns Hopkins University, the Joint Institute for Nuclear Astrophysics, the Kavli Institute for Particle Astrophysics and Cosmology, the Korean Scientist Group, the Chinese Academy of Sciences (LAMOST), Los Alamos National Laboratory, the Max-Planck-Institute for Astronomy (MPIA), the Max-Planck-Institute for Astrophysics (MPA), New Mexico State University, Ohio State University, University of Pittsburgh, University of Portsmouth, Princeton University, the United States Naval Observatory, and the University of Washington.

\section{References}

Archibald, E. N., Dunlop, J. S., Hughes, D. H., et al. 2001, MNRAS, 323, 417 Barger, A. J., Cowie, L. L., \& Sanders, D. B. 1999, ApJ, 518, L5

Bergvall, N., Laurikainen, E., \& Aalto, S. 2003, A\&A, 405, 31

Blain, A. W., Smail, I., Ivison, R. J., Kneib, J.-P., \& Frayer, D. T. 2002, Phys. Rep., 369, 111

Boyle, B. J., Cornwell, T. J., Middelberg, E., et al. 2007, MNRAS, 376, 1182

Buat, V., Marcillac, D., Burgarella, D., et al. 2007, A\&A, 469, 19

Casey, S. C. 1991, ApJ, 371, 183

Chapman, S. C., Barger, A. J., Cowie, L. L., et al. 2003a, ApJ, 585, 57

Chapman, S. C., Blain, A. W., Ivison, R. J., \& Smail, I. R. 2003b, Nature, 422, 695

Chapman, S. C., Blain, A. W., Smail, I., \& Ivison, R. J. 2005, ApJ, 622, 772

Chary, R., \& Elbaz, D. 2001, ApJ, 556, 562

Chary, R., Dickinson, M. E., Teplitz, H. I., Pope, A., \& Ravindranath, S. 2005, ApJ, 635, 1022

Clements, D. L., Farrah, D., Rowan-Robinson, M., et al. 2005, MNRAS, 363, 229

Combes, F. 2004, New Astron. Rev., 48, 583

Commins, E. D. 2004, New Astron. Rev., 48, 567

Condon, J. J. 1992, ARA\&A, 30, 575

Conselice, C. J., Chapman, S. C., \& Windhorst, R. A. 2003, ApJ, 596, L5

Dey, A., Graham, J. R., Ivison, R. J., et al. 1999, ApJ, 519, 610

di Matteo, P., Combes, F., Melchior, A.-L., \& Semelin, B. 2007, A\&A, 468, 61 Dunne, L., \& Eales, S. A. 2001, MNRAS, 327, 697

Dunne, L., Eales, S., Edmunds, M., et al. 2000, MNRAS, 315, 115

Dunne, L., Eales, S., Ivison, R., Morgan, H., \& Edmunds, M. 2003, Nature, 424, 285

Draine, B. T., \& Lee, H. M. 1984, ApJ, 285, 89

Farrah, D., Fox, M., Rowan-Robinson, M., Clements, D., \& Afonso, J. 2004, ApJ, 603, 489

Greve, T. R., Bertoldi, F., Smail, I., et al. 2005, MNRAS, 359, 1165 
Hamuy, M., Phillips, M. M., Suntzeff, N. B., et al. 1996, AJ, 112, 2438

Hildebrand, R. H. 1983, QJRAS, 24, 267

Hughes, D. H., Serjeant, S., Dunlop, J., et al. 1998, Nature, 394, 241

James, A., Dunne, L., Eales, S., \& Edmunds, M. G. 2002, MNRAS, 335, 753

Kauffmann, G., Heckman, T. M., White, S. D. M., et al. 2003, MNRAS, 341, 33

Klaas, U., Haas, M., Müller, S. A. H., et al. 2001, A\&A, 379, 823

Leibundgut, B. 2001, ARA\&A, 39, 67

Lenc, E., \& Tingay, S. J. 2006, AJ, 132, 1333

Lonsdale, C. J., Diamond, P. J., Thrall, H., Smith, H. E., \& Lonsdale, C. J. 2006 ApJ, 647, 185

Madau, P., Ferguson, H. C., Dickinson, M. E., et al. 1996, MNRAS, 283, 1388

Madau, P., Pozzetti, L., \& Dickinson, M. 1998, ApJ, 498, 106

Mattila, S., \& Meikle, W. P. S. 2001, MNRAS, 324, 325

Mattila, S., Väisänen, P., Farrah, D., et al. 2007, ApJ, 659, L9

Meikle, W. P. S., Mattila, S., Pastorello, A., et al. 2007, ApJ, 665, 608

Melchior, A.-L., \& Combes, F. 2007, MNRAS, 381, 1508
Neri, R., Genzel, R., Ivison, R. J., et al. 2003, ApJ, 597, L113

Pen, U. 1999, ApJS, 120, 49

Riess, A. G., Press, W. H., \& Kirshner, R. P. 1996, ApJ, 473, 88

Riess, A. G., Strolger, L.-G., Tonry, J., et al. 2004, ApJ, 607, 665

Riess, A. G., Li, W., Stetson, P. B., et al. 2005, ApJ, 627, 579

Riess, A. G., Strolger, L.-G., Casertano, S., et al. 2007, ApJ, 659, 98

Rowan-Robinson, M. 2003, MNRAS, 344, 13

Sanders, D. B., \& Mirabel, I. F. 1996, ARA\&A, 34, 749

Seaquist, E., Yao, L., Dunne, L., \& Cameron, H. 2004, MNRAS, 349, 1428

Smail, I., Ivison, R. J., Owen, F. N., Blain, A. W., \& Kneib, J.-P. 2000, ApJ, 528, 612

Tacconi, L. J., Neri, R., Chapman, S. C., et al. 2006, ApJ, 640, 228

Tonry, J. L., Schmidt, B. P., Barris, B., et al. 2003, ApJ, 594, 1

Totani, T., \& Kobayashi, C. 1999, ApJ, 526, L65

Vlahakis, C., Eales, S., \& Dunne, L. 2007, MNRAS, 379, 1042

Zylka, R. 1998, MOPSI Users Manual, IRAM, Grenoble 